\title{
FLORÍSTICA E DIVERSIDADE EM ÁREA ECOTONAL CERRADO-PANTANAL NO MUNICÍPIO DE SANTO ANTÔNIO DO LEVERGER, MATO GROSSO, BRASIL
}

\author{
Oacy Eurico de Oliveira ${ }^{1}$, Thelma Shirlen Soares ${ }^{2}$, Reginaldo Brito da Costa $^{3}$, Alberto Dorval $^{3}$, \\ Diego Tyzska Martinez ${ }^{3}$

\footnotetext{
${ }^{1}$ Mestrando do Programa de Pós Graduação em Ciências Florestais e Ambientais, FENF/UFMT. Av. Fernando Correa, ${ }^{\circ} 2367$ 78060-900, Cuiabá, MT

${ }^{2}$ Departamento de Engenharia Florestal - Campus Jataí, UFG-GO. E-mail: thelmasoares@ terra.com.br

${ }^{3}$ Departamento de Engenharia Florestal e Programa de Pós Graduação em Ciências Florestais e Ambientais - FENF, UFMT. Av. Fernando Correa,
} \\ n 2367, CEP 78060-900, Cuiabá, MT. E-mails: reg.brito.costa@ gmail.com; a.dorval@ hotmail.com e diegotyszka@ hotmail.com
}

\section{RESUMO}

O presente estudo teve como objetivo avaliar a composição florística e estrutura do componente arbóreo-arbustivo em área ecotonal de cerrado e pantanal, no município de Santo Antônio do Leverger, MT, na área da cabeceira do rio Caeté, Bacia do Prata. Foram instaladas vinte cinco parcelas de 20 × $20 \mathrm{~m}$, perfazendo área amostral de 1,0 ha. Todos os indivíduos com $\mathrm{CAP} \geq 15,00 \mathrm{~cm}(1,30 \mathrm{~m}$ do solo) foram inventariados. Contabilizaram-se 38 famílias, 71 gêneros e 83 espécies. A família Fabaceae com 15 espécies destacou-se das demais com maior diversidade de espécies. O maior Índice de Valor de Importância foi de Xylopia aromatica. A área basal encontrada de $29,3 \mathrm{~m}^{2} \cdot \mathrm{ha}^{-1}$, pode ser considerada expressiva para a fitofisionomia da área de abrangência do estudo. $\mathrm{O}$ índice de Shannon-Weaver e a equitabilidade de Pielou obtidos foram 3,74 e 0,84, respectivamente, evidenciando uma comunidade com alta diversidade de espécies na área estudada.

Palavras-chave: fragmento florestal, fitossociologia, cerrado, pantanal

\section{FLORISTICS AND DIVERSITY IN A CERRADO-PANTANAL ECOTONAL AREA IN SANTO ANTÔNIO DO LEVERGER, MATO GROSSO STATE, BRASIL}

\section{ABSTRACT}

The assessment aimed to evaluate floristic composition as well as tree and bush in ecotone area of Cerrado and Pantanal, in Santo Antônio do Leverger, Mato Grosso State, Brazil, carried out at the spring stream of the Caeté River, in the Prata River Basin. Twenty five parcels of $20 \mathrm{x}$ $20 \mathrm{~m}$, with sampling area of 1.0 ha were installed. All the individuals with $\mathrm{CBH} \geq 15.00 \mathrm{~cm}(1.30$ $\mathrm{m}$ from the ground) were registered. A total of 38 families, 71 genera and 83 species were identified. The Fabaceae Family, with 15 species, presented the highest diversity when compared to the other plant species. The species Xylopia aromatica showed the highest Importance Value Index. The basal area of $29.3 \mathrm{~m}^{2} . \mathrm{ha}^{-1}$ can be considered expressive for the type of vegetation studied. The Shannon-Weaver and Pielou equitability indexes were 3.74 and 0.84 , respectively, showing a highly diverse community in the studied area.

Keywords: forest fragment, phytosociology, savanna, pantanal 


\section{INTRODUÇÃO}

O Cerrado é o segundo maior bioma brasileiro, localizado em sua maior parte no Brasil central, com área $2.036 .448 \mathrm{~km}^{2}$ correspondendo a $23,92 \%$ da área total do Brasil (IBGE, 2004) e o Pantanal é uma grande planície alagável que cobre aproximadamente $140.000 \mathrm{~km}^{2}$ na bacia do rio Paraguai (GUARIM et al., 2000).

A pressão dos programas de desenvolvimento regional sobre as florestas tropicais, savanas e áreas úmidas acabam ameaçando a integridade biológica de ecossistemas únicos, a exemplo o Pantanal (COSTA \& SCARIOT, 2003).

Salis et al. (2006) afirmam que as primeiras informações sobre a flora pantaneira foram referenciadas no final do século XIX por naturalistas europeus, como Moore em 1895 e Malme em 1905, que trataram as espécies de algumas famílias botânicas, especificamente, Leguminosae e Vochysiaceae.

A caracterização da tipologia vegetal do cerrado para a planície pantaneira tem sido descrita por Marimon \& Lima (2001), Borges \& Shepherd (2005), entre outros.

Por meio do estudo da composição florística e da estrutura fitossociológica de uma floresta, pode-se construir uma base teórica para subsidiar projetos de conservação de recursos genéticos, conservação de áreas similares e recuperação de áreas ou fragmentos florestais degradados, contribuindo para seu manejo (VILELA et al., 1993; CUSTÓDIO FILHO et al., 1994; DRUMOND et al., 1996).

O presente estudo teve como objetivo avaliar a composição florística e estrutura do componente arbóreo-arbustivo em área ecotonal de cerrado e pantanal, no município de Santo Antônio do Leverger, MT.

\section{MATERIAL E MÉTODOS}

A área de estudo, medindo 74 ha, pertence ao Instituto Federal de Educação,
Ciência e Tecnologia de Mato Grosso-IFMT, Campus São Vicente, município de Santo Antônio do Leverger - MT, localizada nas coordenadas geográficas: $15^{\circ} 49^{\prime} 02,3^{\prime \prime} \mathrm{S}$ e $55^{\circ} 24^{\prime} 08,7^{\prime \prime} \mathrm{W}$. Apresenta altitude média de $750 \mathrm{~m}$ acima do nível do mar, clima do tipo C2 wA'a', classificado por Thornthwaite (1948) como sub-úmido, com índice hídrico de 0 a 20, moderada deficiência de água no inverno e evapotranspiração superior a 1.140 $\mathrm{mm} \cdot \mathrm{ano}^{-1}$. Seu clima apresenta duas estações distintas e bem definidas, uma chuvosa, de outubro a abril, e outra seca, de maio a setembro (MESQUITA et al., 2013).

A formação vegetal predominante é o cerrado (desde campo limpo até cerradão), apresentando floresta decídua na encosta dos morros e floresta de galeria ao longo dos rios, além de, em alguns trechos, vegetação típica de áreas alagadas (AMOROZO, 2002).

Os solos são classificados como Podzólico Vermelho Escuro Distrófico (Argissolo Vermelho Distrófico) e Latossolo Vermelho Escuro Distrófico (Latossolo Vermelho Distrófico) associados às Areias Quartzosas, que se relacionam especialmente às coberturas terciárias desenvolvidas sobre arenito de formação furnas, com relevo plano a suave ondulado (BRASIL, 1982).

Para a avaliação qualitativa e quantitativa dos indivíduos arbóreos e arbustivos da vegetação, foi utilizado o método de parcelas (MÜELLER-DOMBOIS \& ELLENBERG, 1974). Foram alocadas vinte e cinco parcelas fixas de $20 \mathrm{~m}$ x $20 \mathrm{~m}$ $\left(400 \mathrm{~m}^{2}\right)$, totalizando área amostral de $10.000 \mathrm{~m}^{2}$ (1,0 ha). No interior das parcelas foram inventariados os indivíduos arbóreos e arbustivos (exceto as lianas), com circunferência à altura do peito $(\mathrm{CAP}) \geq 15$ $\mathrm{cm}$, mensurados com fita métrica a $1,30 \mathrm{~m}$ do solo, e estimada a altura com vara de 6 metros. A amostragem foi realizada entre os meses de abril e outubro de 2010.

A identificação taxonômica das espécies ocorreu mediante consultas a 
herbários, especialistas e por meio de literatura especializada. A sinonímia e grafia dos taxons foram atualizadas mediante consulta ao índice de espécies do Royal Botanic Garden e do banco de dados do Missouri Botanical Garden (MOBOT, 2009).

O sistema de classificação taxonômica adotado foi o APG III (2009), mais utilizado atualmente. $\mathrm{O}$ material botânico coletado em estado reprodutivo foi analisado, catalogado e armazenado no Herbário Central da Universidade Federal de Mato Grosso.

Para caracterização florística da área, foram utilizados os índices de ShannonWeaver (H') e equitabilidade de Pielou (J'). Para descrever a estrutura fitossociológica da comunidade arbórea e arbustiva foram estimados, para espécies e famílias, os parâmetros: Densidade absoluta (DAi), Densidade relativa (DRi), Frequência absoluta (FA), Frequência relativa (FR), Dominância absoluta (DoA), Dominância relativa (DoR), Índice de valor de cobertura (IVC) e Índice de valor de importância (IVI) (MÜELLER-DOMBOIS \& ELLENBERG, 1974).

\section{RESULTADOS E DISCUSSÃO}

A riqueza florística e a diversidade na área estudada foram de 38 famílias, 71 gêneros, 83 espécies (Tabela 1) e um táxon não identificado. Valores similares foram encontrados por Salis et al. (2006), que encontraram 37 famílias, 74 gêneros e 86 espécies, analisando diferentes áreas no pantanal de Mato Grosso do Sul. O número de famílias e espécies foram superiores ao encontrado por Guarim et al. (2000) e Lehn et al. (2008) em análise de vegetação no pantanal, e superiores aos encontrados por Felfili et al. (2007) em matas de galeria na região de cerrado. Tal diferença está associada a maior diversidade de espécies das áreas ripárias e ao fato de ser uma região de ecótono.
O índice de diversidade de Shannon $\left(H^{\prime}\right)$ encontrado de 3,74, foi superior aos relatados nos estudos de Felfili et al. (2007) em matas de galeria e próximo ao registrado por Borges \& Shepherd (2005), de 3,75, indicando alta diversidade de espécies na área estudada.

O resultado do índice 0,84 para equitabilidade de Pielou ( $\left.\mathrm{J}^{\prime}\right)$, foi superior ao registrado por Moura (2010), na região do pantanal de Cáceres-MT, onde foi encontrado o valor de 0,82 para $\left(\mathrm{J}^{\prime}\right)$, confirmando alta uniformidade nas proporções do número de indivíduos/número de espécies dentro da comunidade florestal estudada.

A área apresentou densidade absoluta de 746 indivíduos.ha ${ }^{-1}$, valores inferiores aos obtidos por Arruda (2007), Borges \& Shepherd (2005), Moura (2010), que registraram 1.024, 1.653 e 993 indivíduos.ha ${ }^{-1}$, respectivamente. Estes valores de menores magnitudes podem estar relacionados à utilização de metodologias $\mathrm{e}$ diâmetros de inclusão diferentes aos adotados neste levantamento ou das condições locais que podem favorecer ou não a densidade e o desenvolvimento das espécies. A área basal foi de 29,3 $\mathrm{m}^{2} \cdot \mathrm{ha}^{-1}$, sendo superior à dominância descrita por Moura (2010) em estudo na região de Cáceres-MT, que obteve $10,33 \mathrm{~m}^{2} \cdot \mathrm{ha}^{-1}$.

As famílias mais ricas em número de espécie foram: Fabaceae com 15 espécies, Moraceae (5 espécies), Annonaceae, Euphorbiaceae e Rubiaceae (4 espécies cada), Combretaceae, Lauraceae, Malvaceae, Myrtaceae e Sapotaceae (3 espécies cada), respondendo por $56,1 \%$ das espécies amostradas (Tabela 1).

As famílias Apocynaceae, Araliaceae, Arecaceae, Bignoniaceae, Boraginaceae, Burseraceae, Chrysobalanaceae, Melastomataceae e Urticaceae apresentaram duas espécies cada. Outras 18 famílias foram representadas por uma única espécie. 
Tabela 1. Relação das famílias e espécies, com seus respectivos nomes populares, número de indivíduos amostrados e sua representatividade na área da cabeceira do Rio Caeté, área ecotonal cerrado-pantanal no município de Santo Antônio do Leverger, MT, 2010 .

\begin{tabular}{|c|c|c|c|c|}
\hline \multirow[b]{2}{*}{ Família/espécie } & \multirow{2}{*}{$\begin{array}{c}\text { Nome } \\
\text { Popular }\end{array}$} & \multirow{2}{*}{$\begin{array}{c}\mathrm{N}^{\circ} \text { de } \\
\text { indivíduos }\end{array}$} & \multicolumn{2}{|c|}{ Representatividade } \\
\hline & & & $\begin{array}{c}\text { Família } \\
(\%)\end{array}$ & $\begin{array}{c}\text { Espécie } \\
(\%)\end{array}$ \\
\hline ANACARDIACEAE & & 2 & 0,27 & \\
\hline Tapirira guianensis Aublet. & Pombeiro & 2 & & 0,27 \\
\hline ANNONACEAE & & 89 & 11,93 & \\
\hline Unonopsis sp. & - & 3 & & 0,4 \\
\hline Unonopsis lindmanii Fries & Muxiba & 5 & & 0,67 \\
\hline Xylopia aromatica (Lam.) Mart. & Pindaíba & 80 & & 10,72 \\
\hline sp.1 & - & 1 & & 0,13 \\
\hline APOCYNACEAE & & 4 & $\mathbf{0 , 5 4}$ & \\
\hline Aspidosperma subincanum Mart. & Guatambu & 1 & & 0,13 \\
\hline Himatanthus sucuuba (Spruce) Woodson & Angélica & 3 & & 0,4 \\
\hline ARALIACEAE & & 12 & 1,61 & \\
\hline $\begin{array}{l}\text { Schefflera morototoni (Aubl.) Maguire, } \\
\text { Steyerm. \& Frodin }\end{array}$ & Mandiocão & 7 & & 0,94 \\
\hline Schefflera vinosa (Jacq.) Lodd. ex Mart. & Mandiocão & 5 & & 0,67 \\
\hline ARECACEAE & & 2 & $\mathbf{0 , 2 7}$ & \\
\hline Acrocomia aculeata (Jacq.) Lodd. ex Mart. & Bocaiúva & 1 & & 0,13 \\
\hline sp. 2 & - & 1 & & 0,13 \\
\hline ASTERACEAE & & 1 & $\mathbf{0 , 1 3}$ & \\
\hline Dasyphyllum brasiliense (Spreng) Cabrera & Espinha-agulha & 1 & & 0,13 \\
\hline BIGNONIACEAE & & 23 & 3,08 & \\
\hline Jacaranda copaia (Aubl.) D. Don & Pinho & 7 & & 0,94 \\
\hline Sparattosperma leucanthum Vell (Schum) & Tarumã & 16 & & 2,14 \\
\hline BORAGINACEAE & & 13 & 1,74 & \\
\hline Cordia alliodora Cham. & Louro-branco & 10 & & 1,34 \\
\hline Cordia glabrata Mart. DC. & Louro-preto & 3 & & 0,4 \\
\hline BURSERACEAE & & 7 & 0,94 & \\
\hline Protium heptaphyllum Aubl. (March) & Amescla & 4 & & 0,54 \\
\hline Protium pilosissimum Engl. & Laranjinha & 3 & & 0,4 \\
\hline CHRYSOBALANACEAE & & 20 & 2,68 & \\
\hline Licania humilis Cham. et Schldl. & Sassafrás & 7 & & 0,94 \\
\hline Licania micrantha Sagote Mig & Pau-terra & 13 & & 1,74 \\
\hline CLUSIACEAE & & 1 & $\mathbf{0 , 1 3}$ & \\
\hline Vismia guianensis (Aubl.) Pers. & Lacre & 1 & & 0,13 \\
\hline COMBRETACEAE & & 5 & $\mathbf{0 , 6 7}$ & \\
\hline Buchenavia grandis Ducke & Tarumarana & 1 & & 0,13 \\
\hline
\end{tabular}


Tabela 1. Continuação...

\begin{tabular}{|c|c|c|c|c|}
\hline \multirow[b]{2}{*}{ Família/espécie } & \multirow{2}{*}{$\begin{array}{c}\text { Nome } \\
\text { popular }\end{array}$} & \multirow{2}{*}{$\begin{array}{c}\mathrm{N}^{\mathbf{o}} \text { de } \\
\text { indivíduos }\end{array}$} & \multicolumn{2}{|c|}{ Representatividade } \\
\hline & & & $\begin{array}{l}\text { Família } \\
(\%)\end{array}$ & $\begin{array}{l}\text { Espécie } \\
(\%)\end{array}$ \\
\hline Buchenavia tomentosa Eichler & Mirindiba & 3 & & 0,4 \\
\hline Terminalia argentea Mart et Succ. & Pau-de-bicho & 1 & & 0,13 \\
\hline CONNARACEAE & & 11 & 1,47 & \\
\hline Connarus perrottetii (DC.) Planch. & Folha-parda-estreita & 11 & & 1,47 \\
\hline EBENACEAE & & 8 & 1,07 & \\
\hline Diospyros brasiliensis Mart. & Olho-de-boi & 8 & & 1,07 \\
\hline ELAEOCARPACEAE & & 17 & 2,28 & \\
\hline Sloanea guianensis (Aubl.) Benth. & Pateiro & 17 & & 2,28 \\
\hline EUPHORBIACEAE & & 5 & 0,67 & \\
\hline Hyeronima alchorneoides Fr. Allem. & Araputanga & 1 & & 0,13 \\
\hline Mabea fistulifera Mart. & Fedegoso & 2 & & 0,27 \\
\hline Maprounea guianensis Aublet & Carobinha & 1 & & 0,13 \\
\hline Sapium glandulatum (Vell.) Pax. & Sarã-de-leite & 1 & & 0,13 \\
\hline FABACEAE & & 171 & 22,92 & \\
\hline Anadenanthera falcata (Benth.) & Angico & 4 & & 0,54 \\
\hline Anadenanthera macrocarpa Benth. & Angico-branco & 2 & & 0,27 \\
\hline Apuleia leiocarpa (Vogel) Macbride) & Garapeira & 2 & & 0,27 \\
\hline Bauhinia ungulata L. & Pata-de-vaca & 2 & & 0,27 \\
\hline Copaifera langsdorffii Desf. & Pau-de-óleo & 2 & & 0,27 \\
\hline Dipteryx alata Vogel & Cumbaru & 2 & & 0,27 \\
\hline Enterolobium schomburgkii Benth. & Farinha-seca & 1 & & 0,13 \\
\hline Enterolobium timbouva Mart. & Orelha-de-macaco & 2 & & 0,27 \\
\hline Hymenaea courbaril L. & Jatobá & 20 & & 2,68 \\
\hline Inga sp. & - & 38 & & 5,09 \\
\hline Inga vera Willd. & Ingá & 30 & & 4,02 \\
\hline Ormosia arborea (Vell.) Harms & Folha-parda & 39 & & 5,23 \\
\hline Platypodium elegans Vog & Canzileiro & 4 & & 0,54 \\
\hline $\begin{array}{l}\text { Samanea tubulosa (Benth.) Barneby } \\
\& \text { J. W. Grimes }\end{array}$ & Bordão-de-velho & 3 & & 0,4 \\
\hline Tachigali aureum Tul. Benth. & Carvoeiro & 20 & & 2,68 \\
\hline LAURACEAE & & 37 & 4,96 & \\
\hline Aniba sp. & - & 11 & & 1,47 \\
\hline sp.3 & - & 1 & & 0,13 \\
\hline Ocotea olivacea A. C. Sm. & Caneleiro & 25 & & 3,35 \\
\hline LYTHRACEAE & & 20 & 2,68 & \\
\hline Physocalymma scaberrimum Pohl & Aricá & 20 & & 2,68 \\
\hline MALPIGHIACEAE & & 11 & 1,47 & \\
\hline Byrsonima spicata (Cav.) DC & Murici & 11 & & 1,47 \\
\hline
\end{tabular}


Tabela 1. Continuação...

\begin{tabular}{|c|c|c|c|c|}
\hline \multirow[b]{2}{*}{ Família/espécie } & \multirow[b]{2}{*}{$\begin{array}{c}\text { Nome } \\
\text { popular }\end{array}$} & \multirow{2}{*}{$\begin{array}{c}\mathbf{N}^{\circ} \text { de } \\
\text { indivíduos }\end{array}$} & \multicolumn{2}{|c|}{ Representatividade } \\
\hline & & & $\begin{array}{c}\text { Família } \\
(\%)\end{array}$ & $\begin{array}{c}\text { Espécie } \\
(\%)\end{array}$ \\
\hline MALVACEAE & & 10 & 1,34 & \\
\hline Guazuma ulmifolia Lam. & Chico-magro & 6 & & 0,8 \\
\hline Apeiba tibourbou Aubl. & Pau-jangada & 2 & & 0,27 \\
\hline Luehea divaricata Mart. & Açoita-cavalo & 2 & & 0,27 \\
\hline MELASTOMATACEAE & & 7 & 0,94 & \\
\hline Bellucia grossularioides (L.) Triana & Jambo & 1 & & 0,13 \\
\hline Miconia cuspidata Naud. & Fedegoso & 6 & & 0,8 \\
\hline MELIACEAE & & 1 & $\mathbf{0 , 1 3}$ & \\
\hline Guarea guidonia (L.) Sleumer) & Marinheiro & 1 & & 0,13 \\
\hline MORACEAE & & 75 & 10,05 & \\
\hline Brosimum rubescens Taub. & Alecrim & 23 & & 3,08 \\
\hline Ficus sp. & - & 1 & & 0,13 \\
\hline Maclura tinctoria $\mathrm{L}$. & Amoreira & 2 & & 0,27 \\
\hline Pseudolmedia laevigata Trécul & Leiteiro & 44 & & 5,9 \\
\hline Pourouma sp. & - & 5 & & 0,67 \\
\hline MYRISTICACEAE & & 10 & 1,34 & \\
\hline Virola sebifera Aubl. & Pimenta-de-macaco & 10 & & 1,34 \\
\hline MYRSINACEAE & & 14 & 1,88 & \\
\hline Rapanea ferruginea (Ruiz et Pav) Mez. & Pururuca & 14 & & 1,88 \\
\hline MYRTACEAE & & 12 & 1,61 & \\
\hline Eugenia sp. & - & 6 & & 0,8 \\
\hline Myrcia fallax (Rich.) DC. & Cascudinho & 1 & & 0,13 \\
\hline Myrcia guianensis O. Berg & Cascudinho & 5 & & 0,67 \\
\hline PIPERACEAE & & 2 & 0,27 & \\
\hline Piper sp. & - & 2 & & 0,27 \\
\hline POLYGONACEAE & & 1 & $\mathbf{0 , 1 3}$ & \\
\hline sp.4 & - & 1 & & 0,13 \\
\hline RHAMNACEAE & & 5 & 0,67 & \\
\hline Rhamnidium elaeocarpum Reissek & Cabriteiro & 5 & & 0,67 \\
\hline RUBIACEAE & & 28 & 3,75 & \\
\hline Cordiera edulis (Rich.) Kuntze & Marmelada & 10 & & 1,34 \\
\hline Cordiera macrophylla (K. Schum.) Kuntze & Marmelada & 10 & & 1,34 \\
\hline Duroia duckei Huber & Marmelada & 7 & & 0,94 \\
\hline Genipa americana $\mathrm{L}$. & Jenipapo & 1 & & 0,13 \\
\hline RUTACEAE & & 2 & 0,27 & \\
\hline Zanthoxylum rhoifolium Lam. & Mama-de-porca & 2 & & 0,27 \\
\hline
\end{tabular}


Tabela 1. Continuação...

\begin{tabular}{|c|c|c|c|c|}
\hline \multirow[b]{2}{*}{ Família/espécie } & \multirow{2}{*}{$\begin{array}{c}\text { Nome } \\
\text { popular }\end{array}$} & \multirow{2}{*}{$\begin{array}{c}\mathrm{N}^{\circ} \mathrm{de} \\
\text { indivíduos }\end{array}$} & \multicolumn{2}{|c|}{ Representatividade } \\
\hline & & & $\begin{array}{c}\text { Família } \\
(\%)\end{array}$ & $\begin{array}{c}\text { Espécie } \\
(\%)\end{array}$ \\
\hline SAPINDACEAE & & 16 & 2,14 & \\
\hline Matayba guianensis Aubl. & Canjarana & 16 & & 2,14 \\
\hline SAPOTACEAE & & 50 & 6,7 & \\
\hline Chrysophyllum gonocarpum (Mart. \& Eichler) Engl. & Rapadura & 3 & & 0,4 \\
\hline Manilkara sp. & - & 4 & & 0,54 \\
\hline Pouteria sp. & - & 43 & & 5,76 \\
\hline SIPARUNACEAE & & 38 & 5,09 & \\
\hline Siparuna guianensis Aublet & Negramina & 38 & & 5,09 \\
\hline URTICACEAE & & 14 & 1,88 & \\
\hline Cecropia hololeuca Miq. & Embaúba & 5 & & 0,67 \\
\hline Cecropia pachystachya Trec. & Embaúba & 9 & & 1,21 \\
\hline VOCHYSIACEAE & & 2 & 0,27 & \\
\hline Qualea grandiflora (Mart.) & Canzileiro & 2 & & 0,27 \\
\hline TOTAL & & 746 & 100 & 100 \\
\hline
\end{tabular}

A família Fabaceae apresentou maior valor da riqueza. Trata-se de uma família bem representativa em levantamentos realizados em outras áreas do pantanal (BORGES \& SHEPHERD, 2005) e em áreas de matas de galeria do bioma cerrado (SILVA JÚNIOR et al., 1998). Seu predomínio pode estar associado ao fato do Planalto Central do Brasil ser um de seus principais centros de diversificação (POLHILL et al., 1981).

As famílias Annonaceae e Rubiaceae estão entre as mais representativas (4 espécies cada), sendo também encontradas em outros trabalhos, como em Borges \& Shepherd (2005).

As famílias de maior representatividade com relação ao número de indivíduos foram: Fabaceae (22,92\%), Annonaceae $(11,93 \%)$, Moraceae $(10,05 \%)$, Sapotaceae $(6,70 \%)$, Siparunaceae $(5,09 \%)$, Lauraceae (4,96\%), Rubiaceae $(3,75 \%)$, Bignoniaceae (3,08\%), Lythraceae e Chrysobalanaceae $(2,68 \%$ cada). A participação dessas famílias no valor da abundância correspondeu a 73,84\%, ou seja, 551 indivíduos da área em estudo (Tabela 1).

É importante notar a presença do gênero Inga, família Fabaceae, ocupando a $6^{a}$ e $7^{a}$ posições, respectivamente. Neste gênero, encontram-se espécies indicadas para serem utilizadas no processo de recuperação de áreas degradadas (MARTINS, 2001). A maioria das espécies é encontrada em formações de diferentes domínios vegetacionais e, por sua ampla distribuição, considerada generalista (MATA \& FELIX, 2007). Este é o caso de Inga vera registrada neste levantamento florístico (Tabela 2).

A porcentagem de espécies que apresentaram apenas um exemplar por hectare foi $2,55 \%$, correspondendo a 18 espécies do total amostrado e pode estar relacionada com o ambiente e o padrão de distribuição espacial. Este resultado é inferior ao encontrado por Daniel \& Arruda (2005), em levantamento da composição florística na região do pantanal de Mato Grosso do Sul, constatando para essa área 
que apenas um indivíduo na amostragem foi equivalente a $38,7 \%$ do total.

Dezoito espécies apresentaram valores do índice de valor de importância (IVI\%) superior a 2\%. Em ordem decrescente: Xylopia aromatica, Hymenaea courbaril, Psedolmedia laevigata, Pouteria sp., Ormosia arborea, Sloanea guianensis, Inga sp., Inga vera, Brosimum rubescens, Ocotea olivacea, Byrsonimia spicata, Siparuna guianensis, Sparattosperma leucanthum, Licania micrantha, Tachigali aureum, Jacaranda copaia, Cordia alliodora e Matayba guianensis, responsáveis por $61,11 \%$ do índice de valor de importância. As 10 espécies com valores mais altos de IVI somaram $45,82 \%$, valores próximos àqueles obtidos por Borges \& Shepherd (2005), que encontraram 49,4\% para as 10 espécies com maiores IVI, demonstrando que algumas delas predominam sobre as demais.

A tendência de um pequeno grupo de espécies despontando sobre os demais tem sido observada em outros levantamentos florísticos (FELFILI \& SILVA JÚNIOR, 1993; FELFILI et al., 1997) e está intimamente ligado às características ecológicas, ao padrão de distribuição espacial e às condições ambientais do local avaliado. $\mathrm{O}$ baixo valor da densidade absoluta apresentado pelas espécies é compensado pelo alto valor de dominância absoluta. Verificou-se que Hymenaea courbaril apresentou valor de densidade relativa quatro vezes menor que Xylopia aromatica (espécie de maior IVI), ocupando a segunda posição por registrar o maior valor de dominância absoluta $\left(4,14 \mathrm{~m}^{2} \cdot \mathrm{ha}^{-1}\right)$.

A família Annonaceae se destacou através de Xylopia aromatica com o maior valor de IVI $(6,55 \%)$ devido a seus elevados valores de densidade e frequência, não sendo superior em termos de densidade de espécies na comunidade (Tabela 2). De forma similar, os autores Borges \& Shepherd (2005), analisando área de cerrado na região, relataram que a família Annonaceae foi a mais representativa em função do grande número de indivíduos por hectare e maior frequência. Este evento, segundo Soares (2009), se deve ao fato desta família apresentar hábito de dispersão zoocórica e frutos com inúmeras sementes, que aumentam as possibilidades de dispersão a longas distâncias.

No que se refere ao índice de valor de cobertura (IVC), as dezoito espécies com valores expressivos com base no índice de valor de importância (IVI), também registraram os maiores valores, representando $65,5 \%$ do total.

Condição semelhante foi observada por Bahia et al. (2009) em vereda preservada na área de proteção ambiental do Rio Pandeiros, Minas Gerais, onde as 10 espécies de maior valor de importância contabilizaram $82,92 \%$ dos indivíduos, sendo também as dez de maior índice de valor de cobertura.

Constatou-se através da distribuição dos indivíduos nas classes de altura, que a maior concentração ocorreu no estrato médio, com $88 \%$ (658) dos indivíduos, seguido pelo estrato superior com $12,0 \%$ (90), diferindo do valor obtido por Daniel \& Arruda (2005), em estudo fitossociológico de área florestal às margens do Rio Dourados, Mato Grosso do Sul, que contabilizou no estrato inferior aproximadamente $47 \%$ dos indivíduos amostrados. Os mesmos autores sugeriram que a concentração dos indivíduos no estrato inferior ocorreu em função da presença de grande número de indivíduos das espécies de sub-bosque. 
Tabela 2. Espécies arbóreas e arbustivas ocorrentes na área da cabeceira Rio Caeté, em área ecotonal cerrado-pantanal por ordem decrescente de IVI. Município de Santo Antônio do Leverger, MT, 2010.

\begin{tabular}{|c|c|c|c|c|c|c|c|c|}
\hline Espécie & $\overline{\mathbf{D A}} \mathbf{i}$ & DRi & DoA & DoR & FA & $\mathbf{F r}$ & IVI \% & IVC \% \\
\hline Xylopia aromatica & 80 & 10,70 & 0,93 & 3,18 & 80 & 5,76 & 6,55 & 6,94 \\
\hline Hymenaea courbaril & 20 & 2,67 & 4,14 & 14,12 & 32 & 2,31 & 6,37 & 8,40 \\
\hline Pseudolmedia laevigata & 44 & 5,88 & 1,69 & 5,78 & 68 & 4,90 & 5,52 & 5,83 \\
\hline Pouteria sp. & 43 & 5,75 & 1,07 & 3,64 & 56 & 4,03 & 4,47 & 4,69 \\
\hline Ormosia arborea & 39 & 5,21 & 0,48 & 1,65 & 60 & 4,32 & 3,73 & 3,43 \\
\hline Sloanea guianensis & 17 & 2,27 & 1,59 & 5,44 & 36 & 2,59 & 3,44 & 3,86 \\
\hline Inga $\mathrm{sp}$. & 38 & 5,08 & 0,71 & 2,43 & 36 & 2,59 & 3,37 & 3,75 \\
\hline Inga vera & 30 & 4,01 & 0,72 & 2,47 & 48 & 3,46 & 3,31 & 3,24 \\
\hline Brosimum rubescens & 23 & 3,07 & 0,80 & 2,73 & 48 & 3,46 & 3,09 & 2,90 \\
\hline Ocotea olivacea & 25 & 3,34 & 0,65 & 2,21 & 48 & 3,46 & 3,00 & 2,78 \\
\hline Byrsonimia spicata & 11 & 1,47 & 1,30 & 4,44 & 36 & 2,59 & 2,84 & 2,96 \\
\hline Siparuna guianensis & 38 & 5,08 & 0,20 & 0,69 & 32 & 2,31 & 2,69 & 2,88 \\
\hline Sparattosperma leucanthum & 16 & 2,14 & 0,91 & 3,12 & 24 & 1,73 & 2,33 & 2,63 \\
\hline Licania micrantha & 13 & 1,74 & 0,89 & 3,05 & 24 & 1,73 & 2,17 & 2,39 \\
\hline Tachigali aureum & 20 & 2,67 & 0,65 & 2,22 & 20 & 1,44 & 2,11 & 2,45 \\
\hline Jacaranda copaia & 7 & 0,94 & 1,05 & 3,60 & 24 & 1,73 & 2,09 & 2,27 \\
\hline Cordia alliodora & 10 & 1,34 & 0,71 & 2,44 & 32 & 2,31 & 2,03 & 1,89 \\
\hline Matayba guianensis & 16 & 2,14 & 0,63 & 2,14 & 24 & 1,73 & 2,00 & 2,14 \\
\hline Physocalymma scaberrimum & 20 & 2,67 & 0,44 & 1,49 & 20 & 1,44 & 1,87 & 2,08 \\
\hline Rapanea ferruginea & 14 & 1,87 & 0,38 & 1,30 & 24 & 1,73 & 1,63 & 1,58 \\
\hline Schefflera vinosa & 5 & 0,67 & 0,83 & 2,84 & 12 & 0,86 & 1,46 & 1,75 \\
\hline Aniba sp. & 11 & 1,47 & 0,23 & 0,78 & 24 & 1,73 & 1,33 & 1,12 \\
\hline Diospyros brasiliensis & 8 & 1,07 & 0,43 & 1,46 & 20 & 1,44 & 1,32 & 1,27 \\
\hline Copaifera langsdorffii & 2 & 0,27 & 0,89 & 3,02 & 8 & 0,58 & 1,29 & 1,65 \\
\hline Virola sebifera & 10 & 1,34 & 0,21 & 0,72 & 24 & 1,73 & 1,26 & 1,03 \\
\hline Connarus perrottetii & 11 & 1,47 & 0,08 & 0,29 & 28 & 2,02 & 1,26 & 0,88 \\
\hline Cordiera edulis & 10 & 1,34 & 0,13 & 0,44 & 24 & 1,73 & 1,17 & 0,89 \\
\hline Schefflera morototoni & 7 & 0,94 & 0,32 & 1,08 & 20 & 1,44 & 1,15 & 1,01 \\
\hline Enterolobium timbouva & 2 & 0,27 & 0,74 & 2,54 & 8 & 0,58 & 1,13 & 1,40 \\
\hline Buchenavia tomentosa & 3 & 0,40 & 0,54 & 1,83 & 12 & 0,86 & 1,03 & 1,12 \\
\hline Cordiera macrophylla & 10 & 1,34 & 0,13 & 0,43 & 16 & 1,15 & 0,97 & 0,88 \\
\hline Cecropia pachystachya & 9 & 1,20 & 0,23 & 0,79 & 12 & 0,86 & 0,95 & 1,00 \\
\hline Manilkara sp. & 4 & 0,53 & 0,32 & 1,08 & 16 & 1,15 & 0,92 & 0,81 \\
\hline Platypodium elegans & 4 & 0,53 & 0,46 & 1,59 & 8 & 0,58 & 0,90 & 1,06 \\
\hline Miconia cuspidata & 6 & 0,80 & 0,20 & 0,67 & 16 & 1,15 & 0,88 & 0,74 \\
\hline Licania humilis & 7 & 0,94 & 0,07 & 0,24 & 20 & 1,44 & 0,87 & 0,59 \\
\hline Eugenia sp. & 6 & 0,80 & 0,09 & 0,32 & 20 & 1,44 & 0,85 & 0,56 \\
\hline Guazuma ulmifolia & 6 & 0,80 & 0,09 & 0,31 & 20 & 1,44 & 0,85 & 0,55 \\
\hline Duroia duckei & 7 & 0,94 & 0,05 & 0,16 & 20 & 1,44 & 0,85 & 0,55 \\
\hline
\end{tabular}


Tabela 2. Continuação...

\begin{tabular}{|c|c|c|c|c|c|c|c|c|}
\hline Espécie & DAi & DRi & DoA & DoR & FA & Fr & IVI \% & IVC \% \\
\hline Chrysophyllum gonocarpum & 3 & 0,40 & 0,34 & 1,15 & 12 & 0,86 & 0,80 & 0,77 \\
\hline Cecropia hololeuca & 5 & 0,67 & 0,14 & 0,48 & 16 & 1,15 & 0,77 & 0,58 \\
\hline Myrcia guianensis & 5 & 0,67 & 0,23 & 0,80 & 8 & 0,58 & 0,68 & 0,73 \\
\hline Unonopsis sp. & 3 & 0,40 & 0,22 & 0,75 & 12 & 0,86 & 0,67 & 0,58 \\
\hline Rhamnidium elaeocarpum & 5 & 0,67 & 0,08 & 0,26 & 12 & 0,86 & 0,60 & 0,46 \\
\hline Protium heptaphyllum & 4 & 0,53 & 0,11 & 0,36 & 12 & 0,86 & 0,59 & 0,45 \\
\hline Unonopsis lindmanii & 5 & 0,67 & 0,03 & 0,11 & 12 & 0,86 & 0,55 & 0,39 \\
\hline Protium pilosissimum & 3 & 0,40 & 0,09 & 0,31 & 12 & 0,86 & 0,53 & 0,36 \\
\hline Cordia glabrata & 3 & 0,40 & 0,07 & 0,25 & 12 & 0,86 & 0,50 & 0,32 \\
\hline Hyeronima alchorneoides & 1 & 0,13 & 0,29 & 1,00 & 4 & 0,29 & 0,47 & 0,57 \\
\hline Anadenanthera falcata & 4 & 0,53 & 0,08 & 0,29 & 8 & 0,58 & 0,47 & 0,41 \\
\hline Pourouma sp. & 5 & 0,67 & 0,04 & 0,12 & 8 & 0,58 & 0,46 & 0,40 \\
\hline Samanea tubulosa & 3 & 0,40 & 0,11 & 0,36 & 8 & 0,58 & 0,45 & 0,38 \\
\hline Enterolobium schomburgkii & 1 & 0,13 & 0,26 & 0,88 & 4 & 0,29 & 0,43 & 0,51 \\
\hline Apuleia leiocarpa & 2 & 0,27 & 0,12 & 0,40 & 8 & 0,58 & 0,42 & 0,34 \\
\hline Qualea grandiflora & 2 & 0,27 & 0,08 & 0,26 & 8 & 0,58 & 0,37 & 0,26 \\
\hline Anadenanthera macrocarpa & 2 & 0,27 & 0,06 & 0,20 & 8 & 0,58 & 0,35 & 0,23 \\
\hline Maclura tinctoria & 2 & 0,27 & 0,06 & 0,20 & 8 & 0,58 & 0,35 & 0,23 \\
\hline Tapirira guianensis & 2 & 0,27 & 0,05 & 0,18 & 8 & 0,58 & 0,34 & 0,22 \\
\hline Mabea fistulifera & 2 & 0,27 & 0,03 & 0,10 & 8 & 0,58 & 0,32 & 0,19 \\
\hline Apeiba tibourbou & 2 & 0,27 & 0,11 & 0,36 & 4 & 0,29 & 0,30 & 0,31 \\
\hline Ficus sp. & 1 & 0,13 & 0,12 & 0,40 & 4 & 0,29 & 0,28 & 0,27 \\
\hline Genipa americana & 1 & 0,13 & 0,11 & 0,37 & 4 & 0,29 & 0,26 & 0,25 \\
\hline Himatanthus sucuuba & 3 & 0,40 & 0,03 & 0,09 & 4 & 0,29 & 0,26 & 0,24 \\
\hline Dipteryx alata & 2 & 0,27 & 0,06 & 0,22 & 4 & 0,29 & 0,26 & 0,24 \\
\hline Terminalia argentea & 1 & 0,13 & 0,10 & 0,35 & 4 & 0,29 & 0,26 & 0,24 \\
\hline Luehea divaricata & 2 & 0,27 & 0,03 & 0,09 & 4 & 0,29 & 0,21 & 0,18 \\
\hline Zanthoxylum rhoifolium & 2 & 0,27 & 0,02 & 0,08 & 4 & 0,29 & 0,21 & 0,17 \\
\hline Bauhinia ungulata & 2 & 0,27 & 0,02 & 0,06 & 4 & 0,29 & 0,20 & 0,16 \\
\hline Piper sp. & 2 & 0,27 & 0,01 & 0,03 & 4 & 0,29 & 0,19 & 0,15 \\
\hline Acrocomia aculeata & 1 & 0,13 & 0,04 & 0,13 & 4 & 0,29 & 0,18 & 0,13 \\
\hline sp.2 & 1 & 0,13 & 0,03 & 0,11 & 4 & 0,29 & 0,18 & 0,12 \\
\hline sp.3 & 1 & 0,13 & 0,02 & 0,08 & 4 & 0,29 & 0,17 & 0,11 \\
\hline Maprounea guianensis & 1 & 0,13 & 0,01 & 0,04 & 4 & 0,29 & 0,16 & 0,09 \\
\hline Bellucia grossularioides & 1 & 0,13 & 0,01 & 0,04 & 4 & 0,29 & 0,15 & 0,09 \\
\hline sp.1 & 1 & 0,13 & 0,01 & 0,03 & 4 & 0,29 & 0,15 & 0,08 \\
\hline Aspidosperma subincanum & 1 & 0,13 & 0,01 & 0,02 & 4 & 0,29 & 0,15 & 0,08 \\
\hline Buchenavia grandis & 1 & 0,13 & 0,01 & 0,02 & 4 & 0,29 & 0,15 & 0,08 \\
\hline Dasyphyllum brasiliense & 1 & 0,13 & 0,01 & 0,02 & 4 & 0,29 & 0,15 & 0,08 \\
\hline sp.4 & 1 & 0,13 & 0,01 & 0,02 & 4 & 0,29 & 0,15 & 0,08 \\
\hline Vismia guianensis & 1 & 0,13 & 0,01 & 0,02 & 4 & 0,29 & 0,15 & 0,08 \\
\hline
\end{tabular}


Tabela 2. Continuação...

\begin{tabular}{l|c|c|c|c|c|c|c|c}
\hline Espécie & DAi & DRi & DoA & DoR & FA & Fr & IVI \% & IVC \% \\
\hline Guarea guidonia & 1 & 0,13 & 0,01 & 0,02 & 4 & 0,29 & 0,15 & 0,08 \\
Myrcia fallax & 1 & 0,13 & 0,01 & 0,01 & 4 & 0,29 & 0,15 & 0,07 \\
Sapium glandulatum & 1 & 0,13 & 0,01 & 0,01 & 4 & 0,29 & 0,14 & 0,07 \\
\hline TOTAL & 746 & 100,0 & 29,3 & 100,0 & 1388 & 100,0 & 100,0 & 100,0 \\
\hline
\end{tabular}

DAi = densidade absoluta; DoA = dominância absoluta $\left(\mathrm{m}^{2} \cdot \mathrm{ha}^{-1}\right) ; \mathrm{FA}=$ frequência absoluta; DRi $=$ densidade relativa; DoR $=$ dominância relativa $\left(\mathrm{m}^{2} \cdot \mathrm{ha}^{-1}\right) ; \mathrm{Fr}=$ freqüência relativa; IVI = índice de valor de importância; IVI $(\%)$ = índice de valor de importância em porcentagem; IVC = índice de valor de cobertura IVC $(\%)=$ índice de valor de cobertura em percentagem.

Observou-se neste estudo, que não houve representantes no estrato inferior. A ausência de indivíduos nessa classe deve-se ao fato da inclusão apenas daqueles com CAP $\geq 15,00 \mathrm{~cm}$. Nesse sentido, Andrade $e t$ al. (2002) lembraram que geralmente a mortalidade tem maior ocorrência entre os indivíduos mais jovens na comunidade.

A altura média dos indivíduos foi de $10,0 \mathrm{~m}$. Este resultado é superior ao relatado por Andrade et al. (2002), para área de cerrado denso na Reserva Ecológica do IBGE - RECOR - Distrito Federal, onde registraram indivíduos com altura até $9,4 \mathrm{~m}$.

\section{CONCLUSÕES}

A família Fabaceae, com maior valor de riqueza, reflete a diversidade, importância ecológica e adaptativa das suas espécies para a região de abrangência do estudo e de forma coerente com a realidade dos biomas e a literatura pertinente.

$O$ índice de Shannon-Weaver e equitabilidade de Pielou obtidos mostraram uma comunidade com alta diversidade de espécies, que se constitui um fator importante para a conservação biológica regional.

As informações das análises da estrutura horizontal, diversidade e similaridade florística ofereceram subsídios importantes para a caracterização dos componentes arbóreo e arbustivo que ocorrem em área de transição fitofisionômica do cerrado-pantanal.

\section{AGRADECIMENTOS}

Ao Instituto Federal de Ciência, Educação e Tecnologia - IFMT, Campus São Vicente e à equipe de campo pelo apoio logístico durante a coleta de dados.

\section{REFERÊNCIAS BIBLIOGRÁFICAS}

AMOROZO, M.C.M. 2002. Uso e diversidade de plantas medicinais em Santo Antônio do Leverger, MT, Brasil. Acta Botânica Brasilica, v.16, n.2, p.189-203.

ANDRADE， L.A.Z.; FELFILI， J.M.; VIOLATTI, L. 2002. Fitossociologia de uma área de cerrado denso na RECOR IBGE, Brasília-DF. Acta Botânica Brasilica, v.16, n.2, p. 225-240.

APG III - Angiosperm Phylogeny Group. 2009. An update of the Angiosperm Phylogeny Group classification for the orders and families of flowering plants: APG III. Botanical Journal of the Linnean Society, London, v. 161, p. 105-121.

ARRUDA, D.L.O. 2007. Florística e diversidade em um fragmento de floresta estacional semidecidual aluvial em Dourados, MS. Floresta, v. 37, n. 2, p.111.

BAHIA, T.O.; LUZ, G.R.; VELOSO, M.D.M.; NUNES, Y.R.F.; NEVES, W.V.N.; BRAGA, L.L.; LIMA, P.C.V. 2009. Veredas na APA do Rio 
Pandeiros: importância, impactos ambientais e perspectivas. Instituto Estadual de Florestas, v.2, n.3, p. 1-14.

BORGES, H.B.N.; SHEPHERD, G.J. 2005.

Flora e estrutura do estrato lenhoso numa comunidade de Cerrado em Santo Antônio do Leverger, MT, Brasil. Revista Brasileira de Botânica, v. 28, n. 1, p. 61-74.

BRASIL. 1982. Departamento Nacional de Produção Mineral. Levantamento de Recursos Naturais. Folha Cuiabá (SD21). Rio de Janeiro: DNPM. Projeto Radambrasil.

COSTA, R.B.; SCARIOT, A. 2003. A Fragmentação florestal e os recursos genéticos. In: R.B. COSTA (Org.). Fragmentação Florestal e Alternativas de Desenvolvimento Rural na Região Centro-Oeste, Campo Grande: UCDB. p.53-74.

CUSTÓDIO FILHO, A.; FRANCO, G.A. D.C.; DIAS, A.C. 1994. Composição florística de um trecho de floresta pluvial atlântica, em regeneração natural após desmatamento diferenciado em Pariqüera-açu, SP, Brasil. Revista do Instituto Florestal, São Paulo, v. 6, p. 87-98.

DANIEL, O.; ARRUDA, L. 2005. Fitossociologia de um fragmento de Floresta Estacional Semidecidual Aluvial às margens do Rio Dourados, Mato Grosso do Sul, Brasil. Scientia Forestalis, n. 68, p.69-86.

DRUMOND, M.A.; BARROS, N.F.; SOUZA, A.L.; SILVA, A.F.; MEIRA NETO, J.A.A. 1996. Alterações fitossociológicas e edáficas na Mata Atlântica em função das modificações da cobertura vegetal. Revista Árvore, Viçosa, MG, v. 20, n. 4, p. 451-466.

FELFILI, J.M.; SILVA JÚNIOR, M.C. 1993. A comparative study of cerrado (sensu stricto) vegetation in Central Brazil. Journal of Tropical Ecology, v.9, p. 277-289.
FELFILI, J.M.; SILVA JUNIOR, M.C.; REZENDE, A.V.; NOGUEIRA, P.E.; WALTER, B.M.T.; SILVA, M.A.; ENCINAS, J.I. 1997. Comparação florística e fitossociológica do cerrado nas chapadas Pratinha e dos Veadeiros. In: LEITE, L.; SAITO, C.H. (Eds.). Contribuição ao conhecimento ecológico do cerrado, Ed. Universidade de Brasília. Brasília, DF. p. 6-11.

FELFILI, J.M.; REZENDE, A.V.; SILVA JÚNIOR, M.C.; SILVA, P.E.N.; WALTER, B.M.T.; ENCINAS, J.I.; SILVA, M.A. 2007. Fitossociologia da vegetação arbórea. In: FELFILI, J.M.; REZENDE, A.V.; SILVA JÚNIOR, M.C. Biogeografia do bioma cerrado: vegetação e solos da Chapada dos Veadeiros. Brasília: Editora Universidade de Brasília: Finatec. P. 4596.

GUARIM, V.L.M.S.; MORAES, E.C.C.; PRANCE, G.T.; RATTER, J.A. 2000. Inventory of a Mesotrophic Callisthene Cerradão in the Pantanal of Mato Grosso, Brazil. Edinburg Journal of Botany, v.3, p.429-436.

IBGE. 2004. Mapa dos Biomas do Brasil. Diretoria de Geociências, Acesso em: 16 fev. 2010. Disponível em: <www.ibge.gov.br/home/geociencias>.

LEHN, C.R.; ALVES, F.M.; DAMASCENO JUNIOR, G.A. 2008. Florística e Fitossociologia de uma área de cerrado sensu stricto na região da borda oeste do pantanal, Corumbá, MS, Brasil. Pesquisas Botânicas, São Leopoldo: Instituto Anchietano de Pesquisas, n.59, p.129-142.

MARIMON, B.S.; LIMA E.S. 2001. Caracterização fitofisionômica e levantamento florístico preliminar no Pantanal dos Rios das Mortes-Araguaia, Cocalinho, Mato Grosso, Brasil. Acta Botânica Brasilica, v.15, n.2, p.213229. 
MARTINS, S.V. 2001. Recuperação de matas-ciliares. Viçosa: Aprenda Fácil, $146 \mathrm{p}$.

MATA, M.F.; FELIX, L.P. 2007. Flora da Paraíba, Brasil: Inga Mill.(Leguminosaemimosoideae). Revista Brasileira de Biociências, v.5, supl.2, p. 135-137.

MESQUITA, F.L.L.; MARQUES FILHO, E.P.; KARAM, H.A.; ALVALÁ, C.S. 2013. Balanço de radiação no pantanal sul mato-grossense durante a estação seca. Revista Brasileira de Meteorologia, v. 28, n. 1, p. 65-74.

MOBOT. 2009. Missouri Botanical Garden. W. Trópicos. Acesso em: 20 out 2010. Disponível em: <http://mobot.mobot.org >

MOURA, I.O. 2010. Fitogeografia do cerrado rupestre: relações florísticoestruturais e ecológicas de espécies lenhosas. 2010. 247p. Tese (Doutorado em Ecologia) - Universidade de Brasília, DF.

MÜLLER-DOMBOIS, D.; ELLENBERG, H. 1974. Aims and methods in vegetation ecology. New York : Ed. John Wiley \& Sons, 574 p.

POLHILL, R.M.; RAVEN, P.H.; STIRTON, C.H. 1981. Evolution and systematics of the Leguminosae. In: Polhill RM \& Raven PH. (Eds.). Advances in legume systematics. Kew. Royal Botanic Garden, v.1, p.1-34.

SALIS, S.M.; ASSIS, M.; CRISPIM, S.M.A.; CASAGRANDE， J.C. 2006. Distribuição e abundância de espécies arbóreas em cerradões no Pantanal, Estado do Mato Grosso do Sul, Brasil. Revista Brasileira de Botânica, v.29, n.3, p.339-352.

SILVA JÚNIOR, M.C.; FELFILI, J.M.; NOGUEIRA, P. E.; REZENDE, A. V. 1998. Análise florística das matas de galeria no Distrito Federal. In: RIBEIRO, J. F. (ed.) Cerrado: matas de galeria. Planaltina: EMBRAPA-CPAC. p. $53-84$.
SOARES, P. 2009. Levantamento fitossociológico da regeneração natural em reflorestamento misto no município de Cotriguaçu, Estado de Mato Grosso. 2009. 49p. Dissertação (Mestre em Ciências Florestais e Ambientais) - Universidade Federal de Mato Grosso, Cuiabá.

THORNTHWAITE C.W. 1948. An approach toward a rational classification of climate. Geographical Review. V. 38, n. 1, p. 55-94.

VILELA, E.A.; OLIVEIRA FILHO, A.T.; GAVINALES, M.L.; CARVALHO, D. A. 1993. Espécies de matas ciliares com potencial para estudos de revegetação no alto Rio Grande, Sul de Minas. Revista Árvore, v. 17, n. 2, p. 117-128.

Recebido em: 10/07/2012 Aceito para publicação em: 05/09/2013 